\title{
Direct Statistical Simulation of Monte Carlo in the Study of Argon Radiation behind the Front of a Strong Shock Wave
}

\author{
A.L. Kusov, V.Yu. Levashov, G.Ya. Gerasimov, P.V. Kozlov, \\ I.E. Zabelinskii, N.G. Bykova \\ Institute of Mechanics, Lomonosov Moscow State University, \\ Moscow, 119192, Russia \\ kusov_al@mail.ru,vyl69@mail.ru
}

\begin{abstract}
A technique for simulating the excitation of electronic levels, bremsstrahlung, and photoionization in the direct statistical Monte Carlo simulation is described. The comparison of simulation results with known experimental and calculated data are presented.
\end{abstract}

Keywords: argon radiation, Monte Carlo simulation, shock heated gas.

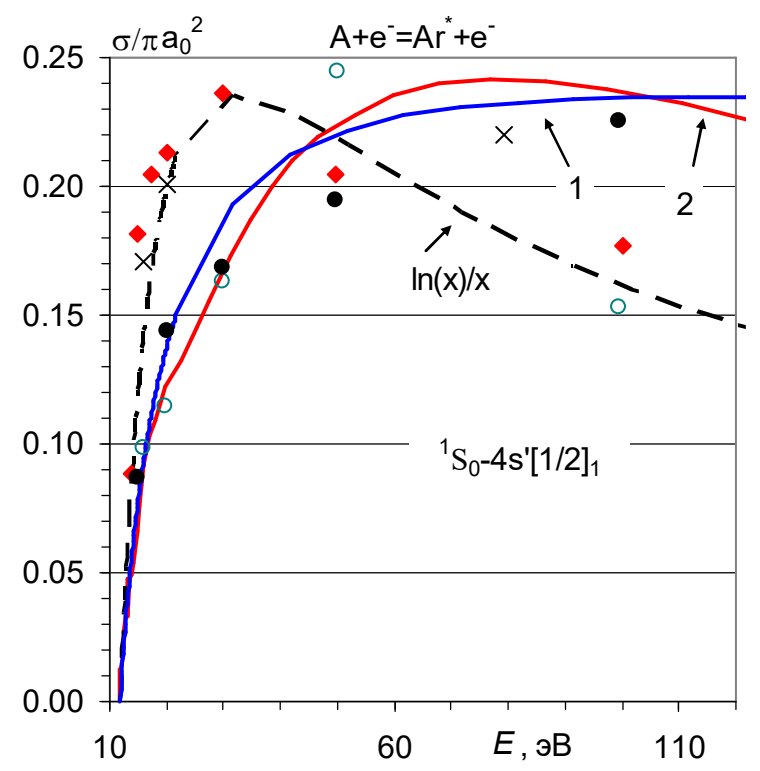

Cross section of an argon atom electronic levels excitation 


\title{
Метод прямого статистического моделирования Монте-Карло при исследовании излучения аргона за фронтом сильной ударной волны
}

\author{
А.Л. Кусов, В.Ю. Левашов, Г.Я. Герасимов, П.В. Козлов, Н.Г. Быкова, \\ И.Е. Забелинский \\ НИИ Механики Московского государственного университета им. М.В. Ломоносова, \\ Россия, Москва, 119192, Мичуринский проспект, 1 \\ kusoval@mail.ru,vyl69@mail.ru
}

\begin{abstract}
Аннотация
Описана методика моделирования возбуждения электронных уровней, тормозного излучения, а также фотоионизации в методе прямого статистического моделирования МонтеКарло. Приведено сравнение результатов моделирования с известными экспериментальными и расчетными данными.
\end{abstract}

Ключевые слова: аргон, излучение, Монте Карло моделирование, ударно нагретый газ.

\section{1. Введение}

Метод прямого статистического моделирования Монте-Карло (ПСМ) основан на построении процесса, который имитирует движение молекул газа и фотонов, столкновение молекул между собой, излучение атомами и молекулами фотонов, взаимодействие молекул и фотонов с границами рассматриваемой области. В методе ПСМ большое количество реальных частиц (молекул, атомов, фотонов) заменяется гораздо меньшим набором модельных частиц. Эти модельные частицы движутся в физическом пространстве, сталкиваются с другими частицами и с твердыми границами аналогично реальной газовой динамике. Столкновения частиц моделируется как мгновенный случайный переход системы из одного состояния в другое, что отличает метод ПСМ от метода молекулярной динамики, в котором столкновения рассматриваются детерминировано. Макроскопические свойства, такие как плотность и средняя скорость, получаются в установившихся потоках путем усреднения по времени [1], [2], [3], [4].

В настоящее время метод ПСМ применяется для моделирования различного рода процессов, в том числе и с учетом различных физико-химических процессов в газовой фазе [5], [6], [7], [8], [9], [10], [11], [12].

Важно, что метод ПСМ позволяет проводить моделирование на элементарном уровне без привлечения законов статистической физики, в том числе, без привлечения понятия температуры. Геометрическое моделирование движения молекул и фотонов не представляет принципиальных трудностей. Поиск пересечения траектории фотона с границами элементарной ячейки расчётной сетки в двумерном и трёхмерном случаях является геометрической задачей. Рассматривается случай малых длин волн, тогда излучение может быть описано в рамках геометрической оптики.

Основную трудность представляют собой рассмотрение столкновений молекул с возбуждением вращательной, колебательной и электронной степеней свободы. 


\section{2. Моделирование процессов в плазме аргона методом Монте-Карло}

\section{1. Моделирование возбуждения электронных уровней энергии}

При температуре газового потока более $10000 \mathrm{~K}$ начинают возбуждаться электронные уровни атомов и молекул. При электронных переходах меняются параметры молекул, поэтому возбуждённая и невозбуждённая молекулы рассматриваются как разные частицы.

Частицы газа (атомы, молекулы и ионы) обладают дискретным набором уровней энергии электронных уровней. Для примера, в таблице 1 приведены значения энергий и статистические веса атома и иона аргона [13], [14].

Средняя энергия электронных уровней частицы согласно распределению Гиббса равна

$$
E=\frac{1}{Z} \sum_{i=1}^{N} e_{i} g_{i} e^{-\frac{E_{i}}{k T}}, \quad Z=\sum_{i=1}^{N} g_{i} e^{-\frac{E_{i}}{k T}}
$$

По данной формуле можно определять равновесную температуру электронных уровней.

Вырождение уровней $g_{i}$ для атомов вычисляется следующим образом:

$$
g_{i}=(2 L+1)(2 S+1)
$$

где $L$ и $S$ - полные орбитальный момент и спин электронов атома. Для молекул с проекциями полного момента количества движения $\Lambda$ и спина $\Sigma$ на межъядерную ось кратность вырождения равна ( $\Lambda$-удвоение)

$$
g_{i}=\left\{\begin{array}{l}
2(2 \Sigma+1), \quad \Lambda>0 \\
2 \Sigma+1, \quad \Lambda=0
\end{array}\right.
$$

В состоянии термодинамического равновесия при заданной температуре $T$ значения энергий частиц определяются согласно общим правилам моделирования случайной величины для дискретной величины [2]. При обмене энергиями в случае использования алгоритма Ларсена - Боргнакке [15] моделирование ничем принципиально не отличается от моделирования колебательной энергии [1]. В литературе отсутствуют формулы, позволяющие ввести среднее время релаксации энергии электронных уровней, что затрудняет применение схем, основанных на использовании алгоритма Ларсена - Боргнакке. Далее рассмотрена кинетическая модель возбуждения электронных уровней.

В простейшей модели сечение возбуждения атома или молекулы электронным ударом согласно [16], [17] определяется как

$$
\sigma_{*}=\pi d_{*}^{2}\left(\frac{\varepsilon}{E_{*}}-1\right),
$$

где $\varepsilon$ - кинетическая энергия налетающего электрона; $E_{*}-$ энергия возбужденного электронного уровня; $d_{*}-$ параметр сечения. То есть предполагается линейный рост сечения при увеличении энергии, что верно при малом превышении энергии $\varepsilon$ над $E_{*}$.

Ч. Пак [18] предложил аппроксимацию для сечения возбуждения в виде

$$
\begin{gathered}
\sigma_{*}=\pi a_{0}^{2}\left(\frac{d_{*}}{a_{0}}\right)^{2} \frac{E_{*}}{\varepsilon} \ln \left(\frac{\varepsilon}{E_{*}}\right) \Theta\left(\varepsilon-E_{*}\right), \\
\sigma_{*}=\pi a_{0}^{2}\left(\frac{d_{*}}{a_{0}}\right)^{2} \frac{\ln x}{x} \Theta(x-1), \quad x=\frac{\varepsilon}{E_{*}},
\end{gathered}
$$


где $d_{*} \sim a_{0}$ - параметр модели (диаметр столкновения); $a_{0}=0.52917706 \AA$ - радиус первой боровской орбиты; $\theta(x)$ - функция Хэвисайда. Данная зависимость вполне адекватно описывает зависимость сечения возбуждения от энергии.

Таблица 1

Таблица 1 - Значения энергий и статистические веса атома и иона аргона, учитываемые в модели

\begin{tabular}{|c|c|c|c|c|c|}
\hline \multicolumn{3}{|c|}{$\mathrm{Ar}$} & \multicolumn{3}{|c|}{$\mathrm{Ar}+$} \\
\hline No. & $E_{i}$, эВ & $g_{i}$ & No. & $E_{i}$, эВ & $g_{i}$ \\
\hline 1 & 0 & 1 & 1 & 0.00 & 4 \\
\hline 2 & 11.548 & 5 & 2 & 0.18 & 2 \\
\hline 3 & 11.624 & 3 & 3 & 13.48 & 2 \\
\hline 4 & 11.723 & 1 & 4 & 16.42 & 20 \\
\hline 5 & 11.828 & 3 & 5 & 17.69 & 28 \\
\hline 6 & 12.907 & 3 & 6 & 17.90 & 6 \\
\hline 7 & 13.076 & 7 & 7 & 18.31 & 12 \\
\hline 8 & 13.095 & 5 & 8 & 18.55 & 14 \\
\hline 9 & 13.153 & 3 & 9 & 18.70 & 10 \\
\hline 10 & 13.172 & 5 & & & \\
\hline 11 & 13.273 & 1 & & & \\
\hline 12 & 13.283 & 3 & & & \\
\hline 13 & 13.302 & 5 & & & \\
\hline 14 & 13.328 & 3 & & & \\
\hline 15 & 13.48 & 1 & & & \\
\hline 16 & 13.845 & 1 & & & \\
\hline 17 & 13.864 & 3 & & & \\
\hline 18 & 13.903 & 5 & & & \\
\hline 19 & 13.979 & 9 & & & \\
\hline 20 & 14.013 & 7 & & & \\
\hline 21 & 14.063 & 5 & & & \\
\hline 22 & 14.068 & 5 & & & \\
\hline 23 & 14.09 & 3 & & & \\
\hline 24 & 14.099 & 7 & & & \\
\hline 25 & 14.153 & 3 & & & \\
\hline 26 & 14.214 & 5 & & & \\
\hline 27 & 14.234 & 5 & & & \\
\hline 28 & 14.236 & 7 & & & \\
\hline 29 & 14.241 & 1 & & & \\
\hline 30 & 14.255 & 3 & & & \\
\hline 31 & 14.304 & 3 & & & \\
\hline 32 & 15.76 & 4 & & & \\
\hline 33 & 15.937 & 2 & & & \\
\hline
\end{tabular}

Для доказательства адекватности формулы Парка, сечение возбуждения электронного уровня атома аргона $4 \mathrm{~s}^{\prime}[1 / 2] 1\left(E_{*}=11.828\right.$ эВ) с основного уровня $1 \mathrm{~S}_{0}$ при столкновении с 
электроном приведены на рис. 1. Маркерами на рисунке обозначены экспериментальные данные [19], кривая 2 - квантовомеханический расчёт [19], прерывистая кривая - по формуле (5), кривая 1 - по формуле (6) с параметрами $\alpha=0.9, \beta=1$.

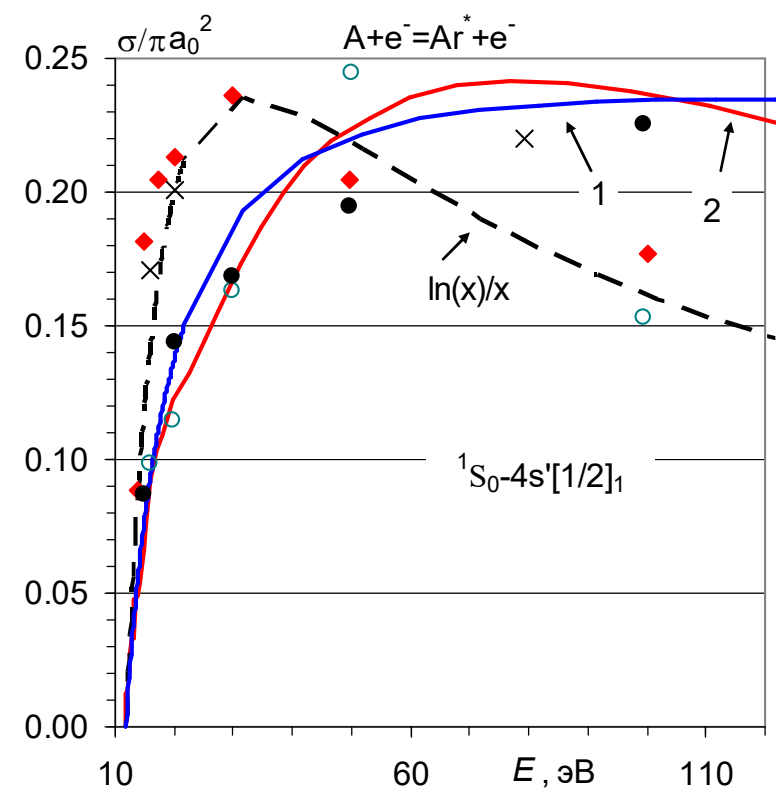

Рис. 1. Сечение возбуждения электронных уровней атома аргона

Модель полной энергии Бёрда (TCE) [1] предполагает несколько другую функцию для сечения реакции, а в данном случае, для возбуждения электронных уровней

$$
\sigma_{*}=\pi a_{0}^{2}\left(\frac{d_{*}}{a_{0}}\right)^{2} \frac{(x-1)^{\alpha}}{x^{\beta}} \Theta(x-1), \quad x=\frac{\varepsilon}{E_{*}},
$$

где $\alpha$ и $\beta$ дополнительные к $d_{*}$ параметры модели.

Допустим, что при столкновении частиц сортов $i$ и $j$ происходит переход между уровнями $|m>\rightarrow| n>$. Уровень $m$ - начальный, а уровень $n$ может быть произвольным, лишь бы хватало энергии на соответствующий переход. Обозначим энергии уровней как $E_{m}$ и $E_{n}$, а кратности вырождения соответственно $g_{m}$ и $g_{n}$. Сечение перехода $\sigma_{i j}^{m \rightarrow n}(\varepsilon)$ должно быть задано либо исходя из экспериментальных данных, либо из квантовомеханических расчётов. Как правило $\sigma_{i j}^{m \rightarrow n}(\varepsilon)$ меньше сечения упругого столкновения, поэтому можно использовать похожий на ТСЕ метод [1]. Вероятность изменения электронного состояния равна

$$
P_{*}=\frac{\sum_{n} \sigma_{i j}^{m \rightarrow n}(\varepsilon)}{\sigma_{i j}\left(E_{t}\right)},
$$

где $\sigma_{i j}\left(E_{t}\right)$ - сечение упругого рассеяния. $\mathrm{C}$ вероятностью $P_{*}$ частица изменит своё электронное состояние. В методе ТСЕ предполагается, что сечения неупругих процессов гораздо меньше сечения упругого столкновения, поэтому число молекулярных столкновений определяется упругим сечением. С учётом формулы Ч. Пака вероятность $P_{*}$ равна

$$
\begin{gathered}
P_{*}=\frac{\pi}{4 \sigma_{i j}\left(E_{t}\right)} \sum_{n}\left(d_{i j}^{m \rightarrow n}\right)^{2} \frac{\ln x_{m \rightarrow n}}{x_{m \rightarrow n}}, \\
x_{m \rightarrow n}= \begin{cases}E_{p} / E_{n}, & m<n, \\
E_{p} / E_{m}, & m>n,\end{cases}
\end{gathered}
$$




$$
E_{p}=E_{t}+E_{m}
$$

где $E_{p}$ - сумма поступательной энергии относительного движения $E_{t}$ и энергии начального уровня $E_{m}$.

Если случайная величина $\gamma$, равномерно распределённая в интервале $(0,1) \gamma<P_{*}$, значит происходит переход на новый уровень $m$ и необходимо определить его случайным образом. Условная ненормированная вероятность перехода (с учётом того, что переход должен осуществиться) на уровень $m$ равна

$$
\begin{gathered}
P_{m \rightarrow n}=\frac{1}{A}\left(d_{i j}^{m \rightarrow n}\right)^{2} \frac{\ln x_{m \rightarrow n}}{x_{m \rightarrow n}}\left(\frac{E_{p}-E_{n}}{E_{p}-E_{m}}\right) \frac{g_{n}}{g_{m}}, \\
A=\sum_{n} P_{m \rightarrow n}
\end{gathered}
$$

Можно легко показать, что при выборе числа с дискретной функцией распределения [2] совсем не обязательно требовать выполнения условия $\sum_{i} P_{i}=1$. Новое значение поступательной энергии относительного движения $E_{t}^{\prime}$ равно

$$
E_{t}^{\prime}=E_{p}-E_{n}
$$

Предварительные результаты численного моделирования показывают, что $d * / a_{0} \sim 1$, более точные значения пока не удалось установить.

Велика вероятность образования возбуждённой частицы при рекомбинации и вообще при химических реакциях, идущих с выделением энергии. Поэтому была введена вероятность что в химической реакции меняются электронные уровни сталкивающихся частиц. Точные значения данных вероятностей также пока установить не удалось.

\section{2. Определение скорости ионизации}

Реакция ионизации имеет вид

$$
\mathrm{A}+\mathrm{M}=\mathrm{A}^{+}+\mathrm{e}^{-}+\mathrm{M}
$$

Эта реакция идентична реакции диссоциации-рекомбинации. Но возникновение заряженных частиц, тем более электронов, обладающих малой массой и большой скоростью, вносит существенные сложности в алгоритм моделирования движения частиц.

В литературе имеются данные о сечениях ионизации с различных уровней [20], [21], [22]. В принципе, в методе ПСМ можно использовать непосредственно данные сечения, однако необходимо сечение рекомбинации, которое можно найти из константы равновесия и сечения ионизации. Поэтому выведем формулу, связывающую сечение ионизации с константой скорости ионизации.

Будем исходить из формулы Г. Бёрда для скорости реакции (6). Если в неё подставить все параметры, то получим зависимость вида

$$
\frac{\sigma_{i}}{\pi a_{0}^{2}}=\left(\frac{d_{i}}{a_{0}}\right)^{2} \frac{(x-1)^{n+\frac{1}{2}+\frac{\xi}{2}}}{x^{1+\frac{\xi}{2}}}, \quad x=\frac{E}{E_{i}},
$$

где $\sigma_{i}$-сечение ионизации; $a_{0}$-радиус Бора; $E$ - энергия столкновения; $E_{i}-$ энергия ионизации; $\xi$ - сумма вращательных и колебательных степеней свободы; $n$ - температурная зависимость в аррениусовской формуле зависимости константы скорости реакции от температуры $K=A T^{n} e^{-E_{i} / k T} ; d_{i}$ - параметр модели. 
Определим скорость ионизации

$$
\begin{gathered}
K_{i}=\int_{E_{i}}^{\infty} \mathrm{d} E_{C} \int_{0}^{\sqrt{2 E_{C} / m_{i j}}}\left(\frac{2 \pi k T}{m_{i j}}\right)^{-3 / 2} \exp \left(-\frac{m_{i j} g^{2}}{2 k T}\right) f_{\text {int }}\left(E_{C}-E_{t r}\right) g \sigma_{i} 4 \pi g^{2} \mathrm{~d} g, \\
f_{\text {int }}(\varepsilon)=\frac{1}{k T \Gamma(\xi / 2)}\left(\frac{\varepsilon}{k T}\right)^{\xi / 2-1} e^{-\frac{\varepsilon}{k T}}, \quad E_{t r}=\frac{1}{2} m_{i j} g^{2}
\end{gathered}
$$

Подставляя все параметры, получаем

$$
\begin{aligned}
K_{i} & =\int_{E_{i}}^{\infty} \sigma_{i} \pi\left(\frac{2}{m_{i j}}\right)^{2}\left(\frac{2 \pi k T}{m_{i j}}\right)^{-3 / 2} \frac{2(k T)^{-\xi / 2}}{\Gamma(\xi / 2)} e^{-\frac{E_{C}}{k T}} \mathrm{~d} E_{C} \int_{0}^{E_{C}}\left(E_{C}-E_{t r}\right)^{\xi / 2-1} E_{t r} \mathrm{~d} E_{t r}= \\
& =\pi a_{0}^{2}\left(\frac{d_{i}}{a_{0}}\right)^{2} \sqrt{\frac{k T}{2 \pi m_{i j}}} \frac{8}{\xi(\xi / 2+1)} \frac{1}{\Gamma(\xi / 2)} e^{-\frac{E_{i}}{k T}}\left(\frac{k T}{E_{i}}\right)^{n-(1 / 2)} \Gamma\left(n+\frac{3}{2}+\frac{\xi}{2}\right)
\end{aligned}
$$

Отдельного рассмотрения требует случай отсутствия внутренних степеней свободы, т.е. $\xi=0$, в этом случае

$$
\begin{aligned}
K_{i} & =\int_{E_{i}}^{\infty} \mathrm{d} E_{C} \int_{0}^{\sqrt{2 E_{C} / m_{i j}}}\left(\frac{2 \pi k T}{m_{i j}}\right)^{-3 / 2} \exp \left(-\frac{m_{i j} g^{2}}{2 k T}\right) f_{\text {int }}\left(E_{C}-E_{t r}\right) g \sigma_{i} 4 \pi g^{2} \mathrm{~d} g= \\
& =\int_{E_{i}}^{\infty} \sigma_{i} \pi\left(\frac{2}{m_{i j}}\right)^{2}\left(\frac{2 \pi k T}{m_{i j}}\right)^{-3 / 2} 2 e^{-\frac{E_{t r}}{k T}} E_{t r} \mathrm{~d} E_{t r}=4 \pi a_{0}^{2}\left(\frac{d_{i}}{a_{0}}\right)^{2} \sqrt{\frac{k T}{2 \pi m_{i j}}} e^{-\frac{E_{i}}{k T}}\left(\frac{k T}{E_{i}}\right)^{n-\frac{1}{2}} \Gamma\left(n+\frac{3}{2}\right)
\end{aligned}
$$

\section{3. Перенос излучения}

Для описания процессов излучения-поглощения для дискретного спектра используют коэффициенты Эйнштейна $A_{n m}$ (коэффициент спонтанного излучения), $B_{n m}$ (коэффициент индуцированного излучения), $B_{m n}$ (коэффициент поглощения). При отсутствии внешнего электромагнитного поля энергия излучения, испускаемая одним атомом при переходе $|n>\rightarrow| m>$ равна [23]

$$
\mathrm{d} E=\mathrm{d} t \int_{4 \pi} A_{n m} \hbar \omega_{n m} \mathrm{~d} \Omega
$$

где $\mathrm{d} \Omega$ - элемент телесного угла. Уровень $m$ - нижний, основной, уровень $n-$ возбуждённый. Это спонтанное излучение, оно изотропно по направлениям. В литературе наряду с $A_{n m}$ используют безразмерную величину, силу осциллятора $f_{n m}$, эти величины пропорциональны друг другу [24]

$$
4 \pi A_{n m}=4 \pi f_{n m} \frac{2 e^{2} \omega^{2}}{m_{e} c^{3}}=4 \pi \frac{4 e^{2} \omega^{3}}{3 \hbar c^{3}} S_{n m}, \quad f_{n m} g_{n}=f_{m n} g_{m},
$$

где $\lambda=2 \pi c / \omega$ - длина волны; $e$ и $m_{e}$ - заряд и масса электрона; $c$ - скорость света; $S_{n m}-$ сила линии перехода $|n>\rightarrow| m>$.

Помимо спонтанного излучения, существует ещё вынужденное излучение. Излучаемая атомом энергия при индуцированном излучении зависит от спектральной интенсивности излучения $I_{v}$

$$
\mathrm{d} E=\mathrm{d} t \int_{4 \pi} B_{n m} I_{v n m} \hbar \omega_{n m} \mathrm{~d} \Omega
$$


Один атом при переходе $|n>\rightarrow| m>$ поглощает энергию, равную

$$
\mathrm{d} E=\mathrm{d} t \int_{4 \pi} B_{m n} I_{v n m} \hbar \omega_{n m} \mathrm{~d} \Omega
$$

\section{4. Уширение атомарных линий}

В квантовой механике показано, что система может иметь как дискретный, так и непрерывный спектр состояний [16], [25]. В случае перехода системы из одного дискретного состояния $\mid n>$ в другое $\mid m>$ излучается фотон с частотой

$$
\hbar \omega_{0}=E_{n}-E_{m}
$$

При этом говорится об атомарной линии или переходе в линии, когда спектр излучения представляет собой дельта-функцию Дирака [25].

Из-за различных процессов, происходящих в газе и плазмы, происходит так называемое уширение линий, когда спектр начинает размазываться по частоте и появляется ширина линии [23]. Все уширения сводят к профилям Гаусса и Лоренца с функцией $p\left(x-x_{0}\right)$, такой, что

$$
\int_{-\infty}^{\infty} p\left(x-x_{0}\right) \mathrm{d} x=1
$$

В этой формуле под $x$ подразумевается обезразмеренная соответствующим образом частота, поэтому физически не может быть $x<0$. Однако ширина линии очень мала и всегда $p(x<0)=0$. Точка $x=x_{0}$ соответствует центру линии.

Штарковское уширение можно также приближённо описать профилем Лоренца.

Профиль Лоренца имеет вид

$$
p\left(x-x_{0}\right)=\frac{a}{\pi\left[a^{2}+\left(x-x_{0}\right)^{2}\right]},
$$

где $a$ параметр. По общим принципам генерирование $x$ с функцией распределения Лоренца проводится следующим образом:

$$
y=\operatorname{tg}\left[\pi\left(\gamma-\frac{1}{2}\right)\right], \quad x=a y+x_{0}
$$

где $\gamma$ - случайная величина, равномерно распределённая в интервале $[0,1]$.

Профиль Гаусса (Gauss) имеет вид

$$
p\left(x-x_{0}\right)=\frac{1}{s \sqrt{\pi}} \exp \left[-\left(\frac{x-x_{0}}{s}\right)^{2}\right]
$$

где $s$ - параметр. Генерирование $x$ с функцией распределения Гаусса проводится, как

$$
r=\sqrt{-\ln \gamma_{1}}, \quad \varphi=2 \pi \gamma_{2}, \quad y=r \cos (\varphi) \quad x=s y+x_{0},
$$

где $\gamma_{1}$ и $\gamma_{2}$ - случайные числа, равномерно распределённые в интервале [0,1].

Можно показать, что

$$
\lim _{a \rightarrow 0} \frac{a}{\pi\left(a^{2}+\left(x-x_{0}\right)^{2}\right)}=\delta\left(x-x_{0}\right), \quad \lim _{s \rightarrow 0} \frac{1}{s \sqrt{\pi}} \exp \left(-\left(\frac{x-x_{0}}{s}\right)^{2}\right)=\delta\left(x-x_{0}\right),
$$


где $\delta\left(x-x_{0}\right)$ - дельта-функция Дирака [24].

В случае статистической независимости двух механизмов уширения $p_{1}(x)$ и $p_{2}(x)$, результирующий профиль $p(x)$ представляется в виде свёртки [25]

$$
p(x)=\int_{-\infty}^{\infty} p_{1}(x-y) p_{2}(y) \mathrm{d} y
$$

В случае совместного действия профилей Лоренца и Гаусса получается профиль Фойгта [27]

$$
\operatorname{Voigt}\left(x-x_{0}, a\right)=p\left(x-x_{0}\right)=\frac{a}{\pi \sqrt{\pi}} \int_{-\infty}^{\infty} \frac{\exp \left(-y^{2}\right) \mathrm{d} y}{a^{2}+\left(x-x_{0}-y\right)^{2}}
$$

Генерирование $x$ c функцией распределения Фойгта проводится, как

$$
\begin{aligned}
& r=\sqrt{-\ln \gamma_{1}}, \quad \varphi=2 \pi \gamma_{2}, \quad y_{G}=r \cos (\varphi), \\
& y_{L}=\operatorname{tg}\left[\pi\left(\gamma_{3}-\frac{1}{2}\right)\right], \quad x=a y_{L}+y_{G}+x_{0}
\end{aligned}
$$

Аппроксимация функции распределения Фойгта имеет вид [28]

$$
\begin{aligned}
W_{g}= & 2 \sqrt{\ln 2}, \quad W_{l}=2 a, \quad S_{d}=\frac{W_{l}-W_{g}}{W_{l}+W_{g}}, \\
W_{v}= & \left\{1-0.18121\left(1-S_{d}^{2}\right)-\sin \left(\pi S_{d}\right) \times\right. \\
& \left.\times\left[0.023665 \exp \left(0.6 S_{d}\right)+0.00418 \exp \left(-1.9 S_{d}\right)\right]\right\}\left(W_{l}+W_{g}\right), \\
\xi=\frac{\left|x-x_{0}\right|}{W_{v}}, \quad W=\frac{W_{l}}{W_{v}}, & \\
\operatorname{Voigt}\left(x-x_{0}, a\right)= & \left\{(1-W) \exp \left(-2.772 \xi^{2}\right)+\frac{W}{1+4 \xi^{2}}+0.016 W(1-W) \times\right. \\
& \left.\times\left[\exp \left(-0.4 \xi^{2.25}\right)-\frac{10}{10+\xi^{2.25}}\right]\right\} \frac{1}{W_{v}\left(1.065+0.447 W+0.058 W^{2}\right)},
\end{aligned}
$$

где $W_{v}-$ полуширина профиля Фойгта.

Максимум функции Фойгта имеет место при $x=x_{0}$ и равен

$$
p_{\max }=\frac{a}{\pi \sqrt{\pi}} \int_{-\infty}^{\infty} \frac{\exp \left(-y^{2}\right) \mathrm{d} y}{a^{2}+y^{2}}=\frac{e^{a^{2}}}{\sqrt{\pi}}
$$

Границу линии $x_{*}$ можно условно принять такой, чтобы $p\left(x_{*}-x_{0}\right)=10^{-3} p_{\max }[28]$

$$
\frac{a}{\pi \sqrt{\pi}} \int_{-\infty}^{\infty} \frac{\exp \left(-y^{2}\right) \mathrm{d} y}{a^{2}+\left(x_{*}-x_{0}-y\right)^{2}}=10^{-3} \frac{e^{a^{2}}}{\sqrt{\pi}},
$$

что даёт для границ линии выражения 


$$
x_{*}=x_{0} \pm \sqrt{\frac{a}{\sqrt{\pi}} 10^{3} e^{-a^{2}}-a^{2}}
$$

Энергия, излучаемая системой атомов с концентрацией $n_{n}$ при переходе $n \rightarrow m$ равна

$$
\frac{\mathrm{d} E}{\mathrm{~d} t}=\oiint_{S}\left(\int_{4 \pi} \int_{-\infty}^{\infty} I_{v}^{\uparrow} \bar{\Omega} \mathrm{d} \Omega \mathrm{d} v\right) \mathrm{d} S=\int_{V} n_{n}\left[\int_{4 \pi} \hbar \omega_{n m}\left(A_{n m}+B_{n m} I_{v n m}\right) \mathrm{d} \Omega\right] \mathrm{d} V,
$$

где $V$ - объём, который занимают поглощающие энергию атомы; $S$ - поверхность этого объёма; $\Omega$ - единичный вектор в направлении элементарного телесного угла $\mathrm{d} \Omega$. С учётом нормировки (21) и формулы Остроградского - Гаусса можно получить выражение для спектральной интенсивности излучения перехода $n \rightarrow m$

$$
\bar{\Omega} \cdot \nabla I_{v}^{\uparrow}=p\left(v-v_{n m}\right) h v_{n m}\left(A_{n m}+B_{n m} I_{v n m}\right)
$$

Энергия, поглощаемая системой атомов с концентрацией $n_{m}$ при переходе $m \rightarrow n$ равна

$$
\frac{\mathrm{d} E}{\mathrm{~d} t}=\oiint_{S}\left(\int_{4 \pi} \int_{-\infty}^{\infty} I_{v}^{\uparrow} \bar{\Omega} \mathrm{d} \Omega \mathrm{d} v\right) \mathrm{d} S=\int_{V}\left(\int_{4 \pi} \int_{-\infty}^{\infty} \kappa_{v} I_{v} \mathrm{~d} \Omega \mathrm{d} v\right) \mathrm{d} V=\int_{V} n_{m}\left(\int_{4 \pi} B_{n m} I_{v n m} \hbar \omega_{n m} \mathrm{~d} \Omega\right) \mathrm{d} V
$$

С учётом нормировки (21)

$$
\bar{\Omega} \cdot \nabla I_{v}^{\uparrow}=\kappa_{v} I_{v}=p\left(v-v_{n m}\right) h v_{n m} B_{n m} I_{v n m} n_{m}
$$

С хорошей точностью в масштабе всего спектрального диапазона

$$
p\left(v-v_{n m}\right) \approx \delta\left(v-v_{n m}\right),
$$

где $\delta(x)$ - дельта-функция Дирака. В этом случае можно приближено записать [24]

$$
I_{v n m} p\left(v-v_{n m}\right) \approx I_{v} p\left(v-v_{n m}\right)
$$

Тогда можно выписать соотношение для истинного коэффициента поглощения для атомарной линии

$$
\kappa_{v}=p\left(v-v_{n m}\right) h v_{n m} B_{m n} n_{m}
$$

В состоянии термодинамического равновесия

$$
\begin{gathered}
I_{v}^{\downarrow}=I_{v}^{\uparrow}=I_{v n m}=B_{v}(T), \\
p\left(v-v_{n m}\right) h v_{n m} B_{m n} I_{v n m} n_{m}=p\left(v-v_{n m}\right) h v_{n m}\left(A_{n m}+B_{n m} I_{v n m}\right)
\end{gathered}
$$

Из этого равенства получаются соотношения для коэффициентов Эйнштейна.

Энергия $Q_{r}$, излучаемая средой в объёме $V$ определяется

$$
Q_{r}=\sum_{m, n} 4 \pi \hbar \omega_{0} B_{m n} n_{m} V\left(1-\exp \left(-\frac{\hbar \omega_{0}}{K T}\right)\right) B_{v}(T)=4 \pi \kappa_{v}^{\prime} B_{v}(T) V,
$$

где $B_{v}(T)$ - равновесная планковская интенсивность излучения; $\omega_{0}$ - частота линии; $n_{m}-$ плотность излучающих атомов.

Энергия фотона несколько отличается от $\hbar \omega_{0}$ из-за наличия уширения и вычисляется из интегрального уравнения 


$$
\begin{gathered}
V_{m}=\sqrt{\frac{2 k T}{m}}, \quad W_{g}=\omega_{0} \frac{V_{m}}{c}, \quad a=\frac{W_{l}}{2 W_{g}}, \quad x_{0}=\frac{c}{V_{m}}, \\
\int_{-\infty}^{\infty} \operatorname{Voigt}\left(y-x_{0}, a\right) \mathrm{d} y=\gamma, \quad \hbar \omega=\hbar \omega_{0} \frac{V_{m}}{c} x,
\end{gathered}
$$

где $T$ - температура газовой среды; $k$ - постоянная Больцмана; $m$ - масса излучающих атомов; $W_{l} / 2$ - полуширина профиля Лоренца; $W_{g}$ - полуширина при доплеровском механизме уширения (полуширина профиля Гаусса); $c$ - скорость света; $\gamma$ - случайная величина, равномерно распределённая в интервале [0,1]. Начальный “вес” фотона на этапе запуска определяется, как

$$
W_{t}=\frac{Q_{r}}{N_{p h} \hbar \omega},
$$

где $N_{p h}$ - запускаемое число фотонов.

Коэффициент поглощения среды с учётом спектрального распределения энергии фотона равен

$$
\begin{gathered}
V_{m}=\sqrt{\frac{2 k T}{m}}, \quad W_{g}=\omega_{m n} \frac{V_{m}}{c}, \quad a=\frac{W_{l}}{2 W_{g}}, \quad x_{0}=\frac{c}{V_{m}}, \quad x=\frac{\omega}{\omega_{n m}} \frac{c}{V_{m}}, \\
\kappa_{\kappa}=\sum_{n, m} \hbar \omega_{m n} B_{m n} n_{m} \operatorname{Voigt}\left(y-x_{0}, a\right)\left[1-\exp \left(-\frac{\hbar \omega_{m n}}{K T}\right)\right]
\end{gathered}
$$

В литературе рассмотрены различные механизмы уширения и сдвига атомарных линий [16], [24], [26], [29], [30], [31], такие как: эффект Доплера, уширение ван Дер Вальса, резонансное уширение, штарковское уширение.

\section{5. Моделирование тормозного излучения}

Свободный электрон не может испустить фотон, так как при этом невозможно удовлетворить одновременно законам сохранения импульса и энергии. Поэтому для реализации такого процесса нужно третья частица, которой может быть ион или нейтральный атом. Схема реакции выглядит следующим образом

$$
\begin{gathered}
e^{-}(\bar{v})+A \rightarrow e^{-}(\vec{v})+A+p h, \\
e^{-}(\bar{v})+A^{+} \rightarrow e^{-}(\vec{v})+A^{+}+p h,
\end{gathered}
$$

где $p h$ означает фотон; $e^{-}$- электрон; $A$ - нейтральный атом; $A^{+}-$ион. При этом должен выполняться закон сохранения энергии

$$
\frac{1}{2} \mu v^{2}=\frac{1}{2} \mu v^{\prime 2}+\hbar \omega
$$

где $\mu \approx m_{e}-$ приведённая масса сталкивающихся частиц; $\omega$ - частота излучаемого света; импульсом фотона можно пренебречь. Максимально возможная энергия излученного фотона равна $\hbar \omega=0.5 \mu v^{2}$, а минимальная длина волны излучения

$$
\lambda_{\min }=\frac{2 h c}{\mu v^{2}}
$$


Вероятность излучения $P$ фотона одним электроном за время $\mathrm{d} t$ при взаимодействии с ионами согласно формуле Крамерса равна [16], [27]

$$
\begin{gathered}
P=A \mathrm{~d} t=n_{i} g \sigma_{R} \mathrm{~d} t \\
\sigma_{R}=\int \pi a_{0}^{2} \frac{16}{3 \sqrt{3}} \alpha^{3}\left(\frac{Z e^{2}}{\hbar g}\right)^{2} \frac{\mathrm{d} \omega}{\omega}=\int \pi a_{0}^{2} \frac{16}{3 \sqrt{3}} \alpha^{5}\left(\frac{Z c}{g}\right)^{2} \frac{\mathrm{d} \omega}{\omega},
\end{gathered}
$$

где $\alpha$ - постоянная тонкой структуры; $a_{0}$ - радиус Бора; $g$ - относительная скорость сталкивающихся частиц (фактически скорость электрона); $n_{i}-$ концентрация ионов.

Электрон тормозится не только в поле иона, но также и в поле нейтральных частиц. Как показано в работе [16] влияние нейтральных частиц можно учесть через отношение сечений тормозного излучения при рассеянии электрона на ионе $\sigma_{\text {ион }}$ и на нейтральной частице $\sigma_{\text {нейт }}$

$$
\frac{\sigma_{\text {нейт }}}{\sigma_{\text {ион }}}=\frac{\sigma_{t r}}{\pi a_{0}^{2}}\left(\frac{E}{2 I_{H} Z}\right)^{2} \text {, }
$$

где $\sigma_{t r}$ - транспортное сечение рассеяния электрона на нейтральной частице; $a_{0}-$ радиус Бора; $Z$ - заряд иона; $I_{\mathrm{H}}=13.6$ эВ - энергия ионизации атома водорода; $E-$ энергия электрона.

С учётом нейтральных частиц вероятность излучения равна

$$
P=A \mathrm{~d} t=\left[n_{i}+\sum_{j} n_{n j}\left(\frac{\sigma_{\text {нейт }}}{\sigma_{\text {ион }}}\right)_{j}\right] g \sigma_{R} \mathrm{~d} t,
$$

где $n_{n j}-$ плотность нейтральных компонент.

Осталось ещё определить вероятность испускание фотона с определённой длиной волны. Согласно (49) соответствующая функция распределения имеет вид

$$
p(\lambda) \mathrm{d} \lambda=\ln \left(\frac{\lambda_{2}}{\lambda_{1}}\right) \frac{\mathrm{d} \lambda}{\lambda}, \quad \int_{\lambda_{1}}^{\lambda_{2}} p(\lambda) \mathrm{d} \lambda=1,
$$

где $\lambda_{2}$ - правая граница спектрального диапазона, а $\lambda_{1}-$ наибольшее значение между левой границей спектра и $\lambda_{\min }$ из (48). Следуя общим принципам [2] длина волны находится как

$$
\lambda=\lambda_{1}\left(\frac{\lambda_{2}}{\lambda_{1}}\right)^{\gamma}
$$

где $\gamma$ - случайная величина, равномерно распределённая в интервале $(0,1)$.

\section{6. Моделирование фотоионизации и фоторекомбинации}

При столкновении с ионом свободный электрон может перейти в связанное состояние с образованием нейтрального атома или молекулы. Такой процесс называется фоторекомбинацией иона и электрона или кратко просто фоторекомбинацией

$$
e^{-}(\bar{v})+A^{+} \rightarrow A+p h
$$

Обратный процесс образования иона при взаимодействии атома с фотоном называется фотоионизацией

$$
A+p h \rightarrow e^{-}\left(\vec{v}^{\prime}\right)+A^{+}
$$


Описание процесса фоторекомбинации во многом аналогично процессу тормозного излучения. Вероятность излучения при фоторекомбинации в расчёте на один электрон равна

$$
P=A \mathrm{~d} t=n_{i} g \sigma_{C}^{n} \mathrm{~d} t
$$

где $\sigma_{C}^{n}$ - сечение фоторекомбинации атома $A$ на уровень $n$. При этом будем предполагать, что сечение не зависит от уровня энергии иона $A^{+}$. Вероятность процесса фотоионизации в расчёте на один атом определяется как

$$
P=n_{p h} c \sigma_{i}^{n} \mathrm{~d} t\left[1-\exp \left(-\frac{h v}{k T}\right)\right]
$$

где $n_{p h}$ - плотность фотонов; $c$ - скорость света; $\sigma_{i}^{n}$ - сечение фотоионизации атома, находящегося на уровне $n$. Плотность фотонов можно определить, поделив плотность энергии излучения на энергию фотона $\hbar \omega$

$$
n_{p h}=\frac{U_{v} \mathrm{~d} v}{h v}
$$

В литературе имеются данные относительно сечений фотоионизации [29], [22], [32], в основном это квантовомеханические расчёты. Для получения данных относительно сечений фоторекомбинации, необходимо найти связь между двумя этими сечениями. Приравняв в состоянии равновесия скорости прямого и обратного процессов, получаем

$$
f(\bar{g}) 4 \pi g^{2} \mathrm{~d} g n_{i} g \sigma_{C}^{n} \mathrm{~d} t n_{e} V=\frac{U_{v}}{h v} c \sigma_{i}^{n} \mathrm{~d} t n_{a}^{n} V \mathrm{~d} v\left[1-\exp \left(-\frac{h v}{k T}\right)\right],
$$

где $n_{a}^{n}$ - число атомов на уровне $n ; V$ - объём занимаемый газом. Для отношения сечений получаем

$$
\frac{\sigma_{i}^{n}}{\sigma_{C}^{n}}=\frac{n_{i} n_{e}}{n_{a}^{n}} \frac{g}{c} \frac{h v}{U_{v}} \frac{f(\bar{g}) 4 \pi g^{2} \mathrm{~d} g}{\mathrm{~d} v} \frac{1}{\left[1-\exp \left(-\frac{h v}{k T}\right)\right]}
$$

Учитывая, что в равновесном случае

$$
U_{v}=\frac{4 \pi}{c} B_{v}, \quad B_{v}=\frac{2 h v^{3}}{c^{2}\left(\exp \left(\frac{h v}{k T}\right)-1\right)}, \quad f_{M}(g)=\left(\frac{m_{e}}{2 \pi k \mathrm{~T}}\right)^{\frac{3}{2}} \exp \left(-\frac{m_{e} g^{2}}{2 k T}\right),
$$

получаем

$$
\frac{\sigma_{i}^{n}}{\sigma_{C}^{n}}=\frac{n_{i} n_{e}}{n_{a}^{n}} \frac{h v}{B_{v}} \frac{f(\bar{g}) g^{3} \mathrm{~d} g}{\mathrm{~d} v} \frac{1}{\left[1-\exp \left(-\frac{h v}{k T}\right)\right]}=\frac{n_{i} n_{e}}{n_{a}^{n}} \frac{g^{3} \mathrm{~d} g c^{2}}{2 v^{2} \mathrm{~d} v}\left(\frac{m_{e}}{2 \pi k T}\right)^{3 / 2} \exp \left(-\frac{m_{e} g^{2}}{2 k T}+\frac{h v}{k T}\right)
$$

Если пренебречь движением иона по сравнению со скоростью электрона, из закона сохранения энергии при фотоионизации и фоторекомбинации следует

$$
\frac{1}{2} m_{e} g^{2}+I=E_{n}+h v
$$

где $I$ - потенциал ионизации; $E_{n}$ - энергия уровня $n$ атома. Дифференцируя (63), получаем

$$
m_{e} g \mathrm{~d} g=h \mathrm{~d} v \Rightarrow \frac{\mathrm{d} g}{\mathrm{~d} v}=\frac{h}{m_{e} g}
$$


Концентрация атомов на уровне $n$ согласно распределения Больцмана равна

$$
\frac{n_{a}^{n}}{n_{a}}=\frac{1}{Z_{a}} g_{n} \exp \left(-\frac{E_{n}}{k T}\right), \quad Z_{a}=\sum_{i} g_{i} \exp \left(-\frac{E_{i}}{k T}\right),
$$

где $E_{i}$ и $g_{i}$ - энергия и кратность вырождения $i$-го уровня; $n_{a}-$ плотность атомов; $Z$ - статистическая сумма, энергию в которой условимся отсчитывать относительно основного уровня, т.е. энергия основного уровня $E_{0}=0$. Концентрацию электронов и ионов можно определить по формуле Саха

$$
\frac{n_{i} n_{e}}{n_{a}}=2\left(\frac{2 \pi m_{e} k T}{h^{2}}\right)^{3 / 2} \frac{Z_{i}}{Z_{a}} \exp \left(-\frac{I}{k T}\right)
$$

Для связи сечений получаем

$$
\frac{\sigma_{i}^{n}}{\sigma_{C}^{n}}=\frac{Z_{i}}{g_{n}} \exp \left(\frac{E_{n}}{k T}-\frac{I}{k T}-\frac{m_{e} g^{2}}{2 k T}+\frac{h v}{k T}\right)\left(\frac{m_{e} g c}{h v}\right)^{2}
$$

С учётом закона сохранения энергии, получаем

$$
\frac{\sigma_{i}^{n}}{\sigma_{C}^{n}}=\frac{Z_{i}}{g_{n}}\left(\frac{m_{e} g c}{h v}\right)^{2}
$$

Оба сечения должны зависеть только от состояния взаимодействующих частиц, но не от температуры. В полученной формуле статистическая сумма зависит от температуры, это следствие того, что не рассматривалась зависимость процесса от состояния иона.

\section{7. Тестирование алгоритма}

Для тестирования предложенного алгоритма проведены расчеты распределения плотности и концентрации электронов для скорости ударной волны $U=5529 \mathrm{~m} / \mathrm{c}$, начальном давлении и температуре невозмущенного газа 2.06 Торр и $297.8 \mathrm{~K}$ соответственно. Результаты расчетов представлены на рис. 2 и 3. Символами на рисунках показаны экспериментальные данные [33], тонкой линией - результаты расчетом по радиационно-столкновительной модели [22], сплошной линией - результаты настоящей работы. Из рисунков видно удовлетворительное согласие результатов ПСМ моделирования с экспериментальными данными.

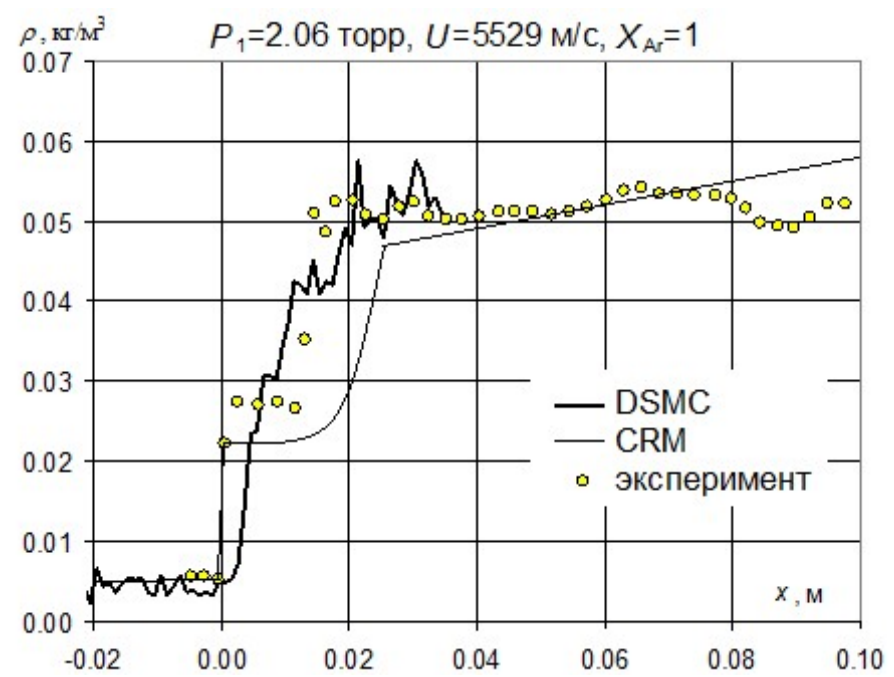

Рис. 2. Распределение плотности в ударной волне, распространяющейся со скоростью $U=5529 \mathrm{~m} / \mathrm{c}$ по аргону при давлении и температуре невозмущенного газа $P=2.06$ Торр и $T=297.8 \mathrm{~K}$ 


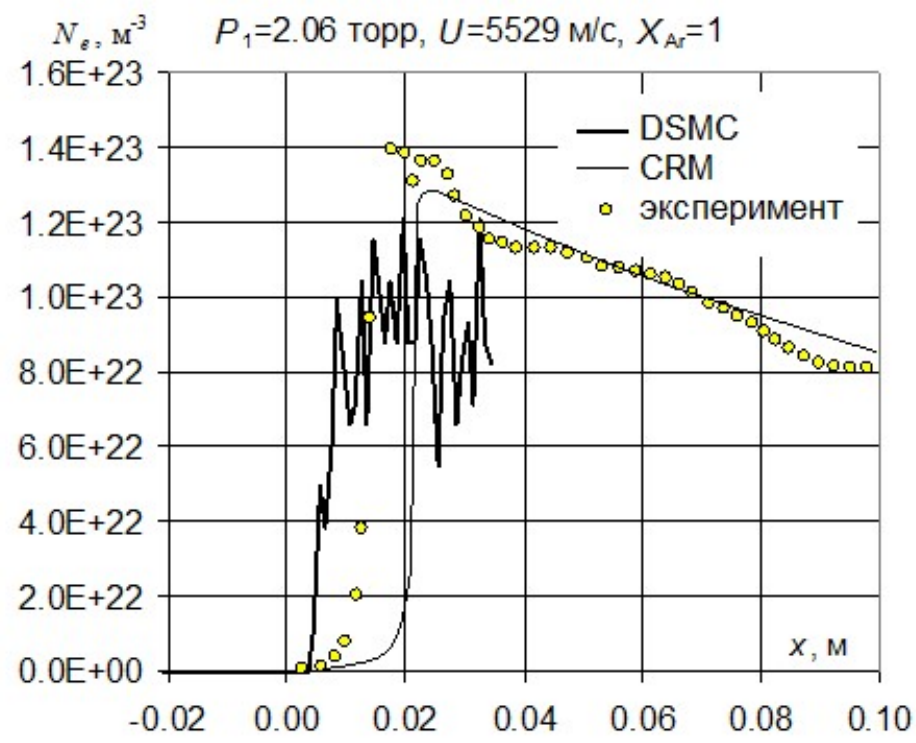

Рис.3. Распределение концентрации электронов в ударной волне, распространяющейся со скоростью $U=5529$ м/с по аргону при давлении и температуре невозмущенного газа $P=2.06$ Торр и $T=297.8 \mathrm{~K}$

Осцилляции в распределении концентраций электронов, представленные на рис. 3 , связаны с тем, что метод ПСМ является статистическим. То есть в результате многократного повторения одного и того же процесса получаются средние значения, например, концентраций компонент или температур. Точное среднее значение получится при бесконечно большом количестве повторений (испытаний), при ограниченном же их числе результат будет получаться в виде последовательности случайных чисел (осцилляций) с определенными математическим ожиданием и дисперсией. Дисперсия $D$ фактически определяет ошибку результата моделирования и уменьшается при увеличении числа испытаний $N$ как $D \sim 1 / \sqrt{N}$.

\section{3. Заключение}

Представлена методика метода ПСМ, которая позволяет проводить расчет структуры ударной волны в аргоне, а также излучения в ней с учетом различных процессов: эволюции заселения возбужденных состояний атома аргона, ионизации, излучения в линиях атома и иона аргона, тормозного излучения, фотоионизации и фоторекомбинации. Предложены модели сечений указанных процессов. Результаты сравнения расчётов и экспериментов показывают, что выбранные модели приводят к качественно правильным результатам, однако параметры моделей нуждаются в дальнейшем уточнении.

\section{Благодарности и ссылки на гранты}

Работа выполнена в соответствии с планом исследований НИИ механики МГУ им. М.В. Ломоносова при финансовой поддержке Российского фонда фундаментальных исследований (проект № 20-08-00343).

\section{Литература}

1. Bird G., Molecular Gas Dynamics and the Direct Simulation of Gas Flows, Oxford. Cl. 1994.

2. Соболь И. М., Численные методы Монте-Карло. Москва: Наука, 1973.

3. Кусов А. Л., “Численное моделирование обтекания цилиндра со сферическим носком методом прямого статистического моделирования Монте-Карло," Математическое моделирование, T. 27, № 12, С. 33-47, 2015. 
4. "Direct Simulation Monte-Carlo (DSMC) Method," in Heat and Mass Transfer, Berlin: Springer Berlin Heidelberg, 2005, pp. 275-315.

5. Dunn S. M. and Anderson J. B., "Direct Monte Carlo simulation of chemical reaction systems: Internal energy transfer and an energy-dependent unimolecular reaction," J. Chem. Phys., vol. 99, no. 9, pp. 6607-6612, 1993.

6. Bruno D., Capitelli M., Esposito F., Longo S., and Minelli P., "Direct simulation of non-equilibrium kinetics under shock conditions in nitrogen," Chem. Phys. Lett., vol. 360, no. 1-2, pp. 31-37, Jul. 2002.

7. Boyd I. D., "Modeling backward chemical rate processes in the direct simulation Monte Carlo method," Phys. Fluids, vol. 19, no. 12, 2007.

8. Shevyrin A. and Bondar Y., "On the calculation of the electron temperature flowfield in the DSMC studies of ionized re-entry flows," Adv. Aerodyn., vol. 2, no. 1, 2020.

9. $\quad$ Fang M., Li Z.-H., Li Z.-H., Liang J., and Zhang Y.-H., "DSMC modeling of rarefied ionization reactions and applications to hypervelocity spacecraft reentry flows," Adv. Aerodyn., vol. 2, no. 1, pp. $1-25,2020$.

10. Bruno D., Capitelli M., Longo S., and Minelli P., "Direct Simulation Monte Carlo Modeling of Non Equilibrium Reacting Flows. Issues for the Inclusion into a ab initia Molecular Processes Simulator," Lect. Notes Comput. Sci. (including Subser. Lect. Notes Artif. Intell. Lect. Notes Bioinformatics), vol. 3044, pp. 383-391, 2004.

11. Кусов А. Л., "Расчёт ионизации методом прямого статистического моделирования Монте-

Карло," //Физико-химическая кинетика в газовой динамике. 2016. Т. 17, вып. 2. http://chemphys.edu.ru/issues/2016-17-2/articles/663/

12. Кусов А. Л., “О возможности моделирования диссоциации кислорода в ударной волне в рамках классических моделей метода прямого статистического моделирования Монте-Карло//Физикохимическая кинетика в газовой динамике. 2016. Т.17, вып. 1. http://chemphys.edu.ru/issues/2016$17-1 /$ articles/612/

13. "Atomic Spectra Database | NIST." [Online]. Available: https://www.nist.gov/pml/atomic-spectradatabase. [Accessed: 25-Nov-2020].

14. Kapper M. G. and Cambier J.-L., "Ionizing shocks in argon. Part I: Collisional-radiative model and steady-state structure," J. Appl. Phys., vol. 109, no. 11, p. 113308, Jun. 2011.

15. Borgnakke C. and Larsen P. S., "Statistical collision model for Monte Carlo simulation of polyatomic gas mixture," J. Comput. Phys., vol. 18, no. 4, pp. 405-420, 1975.

16. Зельдович Я. Б., Райзер Ю. П., Физика ударных волн и высокотемпературных гидродинамических явлений. Москва: Физматлит, 2008.

17. Великович А. Л., Либерман М. А., Физика ударных волн в газах и плазме. Москва: Наука, 1987.

18. Park C., Nonequilibrium hypersonic aerothermodynamics. New York,: John Wiley \& Sons, 1989.

19. Zatsarinny O., Wang Y., and Bartschat K., "Electron-impact excitation of argon at intermediate energies," Phys. Rev. A - At. Mol. Opt. Phys., vol. 89, no. 2, pp. 1-8, 2014.

20. Биберман Л. М., Воробьев В. С., Якубов И. Т., Кинетика неравновесной низкотемпературной плазмы. Москва: Наука, 1982.

21. Bacri J. and Gomes A. M., "Influence of atom-atom collisions on thermal equilibrium in argon arc discharges at atmospheric pressure," J. Phys. D. Appl. Phys., vol. 11, no. 16, pp. 2185-2197, 1978.

22. Kapper M. G. and Cambier J. L., "Ionizing shocks in argon. Part I: Collisional-radiative model and steady-state structure," J. Appl. Phys., vol. 109, no. 11, 2011.

23. Бай Ши-и, Динамика излучающего газа. Москва: Мир, 1968.

24. Пилюгин Н. Н., Тирский Г. А., Динамика ионизованного излучающего газа. Москва: Изд.-во МГУ, 1989.

25. Дирак П. А. М., Принциипы квантовой механики. Москва: Наука, 1979.

26. Грим Г., Спектроскопия плазмы. Москва: Атомиздат, 1969.

27. Собельман И. И., Введение в теорию атомных спектров. Москва: Физ.-мат. лит., 1963. 
28. Arnold J. O., Whiting E. E., and Lyle G. C., "Line by line calculation of spectra from diatomic molecules and atoms assuming a voigt line profile," J. Quant. Spectrosc. Radiat. Transf., vol. 9, no. 6, pp. 775-798, Jun. 1969.

29. Каменщиков В. А., Пластинин Ю. А., Николаев В. М., Новицкий Л. А., Радиационные свойства газов при высоких температурах. Москва: Машиностроение, 1971.

30. Вайнштейн Л. А., Собельман И. И., Юков Е. А., Возбуждение атомов и уширение спектральных линий. Москва: Наука, 1979.

31. Пенер С. С., Количественная и молекулярная спектроскопия и излучательная способность газов. Москва: Изд. Иностранной литературы, 1963.

32. “TOPbase - база данных по сечениям фотоионизации.".

33. Glass I. I. and Liu W. S., "Effects of hydrogen impurities on shock structure and stability in ionizing monatomic gases," J. Fluid Mech., vol. 84, no. 1, pp. 55-77, 1978.

\section{References}

1. Bird, G., Molecular Gas Dynamics and the Direct Simulation of Gas Flows, Oxford. Cl. 1994.

2. Sobol', I. M., Chislennye metody Monte-Karlo, Moskva: Nauka, 1973.

3. Kusov, A. L., "Chislennoe modelirovanie obtekanija cilindra so sfericheskim noskom metodom prjamogo statisticheskogo modelirovanija Monte-Karlo," Matematicheskoe modelirovanie, Vol. 27, No. 12, 2015, pp. 33-47.

4. "Direct Simulation Monte-Carlo (DSMC) Method," in Heat and Mass Transfer, Berlin: Springer Berlin Heidelberg, 2005, pp. 275-315.

5. Dunn, S. M. and Anderson, J. B., "Direct Monte Carlo simulation of chemical reaction systems: Internal energy transfer and an energy-dependent unimolecular reaction," J. Chem. Phys., Vol. 99, No. 9, 1993, pp. 6607-6612.

6. Bruno, D., Capitelli, M., Esposito, F., Longo, S., and Minelli, P., "Direct simulation of non-equilibrium kinetics under shock conditions in nitrogen," Chem. Phys. Lett., Vol. 360, No. 1-2, 2002, pp. 31-37.

7. Boyd, I. D., "Modeling backward chemical rate processes in the direct simulation Monte Carlo method," Phys. Fluids, Vol. 19, No. 12, 2007.

8. Shevyrin, A. and Bondar, Y., "On the calculation of the electron temperature flowfield in the DSMC studies of ionized re-entry flows," Adv. Aerodyn., Vol. 2, No. 1, 2020.

9. Fang, M., Li, Z.-H., Li, Z.-H., Liang, J., and Zhang, Y.-H., "DSMC modeling of rarefied ionization reactions and applications to hypervelocity spacecraft reentry flows," Adv. Aerodyn., Vol. 2, No. 1, 2020, pp. 1-25.

10. Bruno, D., Capitelli, M., Longo, S., and Minelli, P., "Direct Simulation Monte Carlo Modeling of Non Equilibrium Reacting Flows. Issues for the Inclusion into a ab initia Molecular Processes Simulator," Lect. Notes Comput. Sci. (including Subser. Lect. Notes Artif. Intell. Lect. Notes Bioinformatics), Vol. 3044, 2004, pp. 383-391.

11. Kusov, A. L., "Raschjot ionizacii metodom prjamogo statisticheskogo modelirovanija Monte-Karlo," Fiziko-himicheskaja kinetika v gazovoj dinamike (Phisical-Chemical Kinetics in Gas Dynamics), 2016. Vol. 17, No. 2. http://chemphys.edu.ru/issues/2016-17-2/articles/663/.

12. Kusov A. L., "O vozmozhnosti modelirovanija dissociacii kisloroda v udarnoj volne v ramkah klassicheskih modelej metoda prjamogo statisticheskogo modelirovanija Monte-Karlo," Fiziko-himicheskaja kinetika v gazovoj dinamike (Phisical-Chemical Kinetics in Gas Dynamics), Vol. 17, No. 1, 2016, http://chemphys.edu.ru/issues/2016-17-1/articles/612/

13. "Atomic Spectra Database | NIST." [Online]. Available: https://www.nist.gov/pml/atomic-spectradatabase. [Accessed: 25-Nov-2020].

14. Kapper, M. G. and Cambier, J.-L., "Ionizing shocks in argon. Part I: Collisional-radiative model and steady-state structure," J. Appl. Phys., Vol. 109, No. 11, Jun. 2011, p. 113308. 
15. Borgnakke, C. and Larsen, P. S., "Statistical collision model for Monte Carlo simulation of polyatomic gas mixture," J. Comput. Phys., Vol. 18, No. 4, 1975, pp. 405-420.

16. Zel'dovich, Ja. B., Rajzer, Ju. P., Fizika udarnyh voln $i$ vysokotemperaturnyh gidrodinamicheskih javlenij, Moskva: Fizmatlit, 2008.

17. Velikovich, A. L., Liberman, M. A., Fizika udarnyh voln v gazah i plazme, Moskva: Nauka, 1987.

18. Park, C., Nonequilibrium hypersonic aerothermodynamics. New York,: John Wiley \& Sons, 1989.

19. Zatsarinny, O., Wang, Y., and Bartschat, K., "Electron-impact excitation of argon at intermediate energies," Phys. Rev. A - At. Mol. Opt. Phys., Vol. 89, No. 2, 2014, pp. 1-8.

20. Biberman, L. M., Vorob'ev, V. S., Jakubov, I. T., Kinetika neravnovesnoj nizkotemperaturnoj plazmy, Moskva: Nauka, 1982.

21. Bacri, J. and Gomes, A. M., "Influence of atom-atom collisions on thermal equilibrium in argon arc discharges at atmospheric pressure," J. Phys. D. Appl. Phys., Vol. 11, No. 16, 1978, pp. 2185-2197.

22. Kapper, M. G. and Cambier, J. L., "Ionizing shocks in argon. Part I: Collisional-radiative model and steady-state structure,” J. Appl. Phys., Vol. 109, No. 11, 2011.

23. Baj, Shi-i, Dinamika izluchajushhego gaza, Moskva: Mir, 1968.

24. Piljugin, N. N., Tirskij, G. A., Dinamika ionizovannogo izluchajushhego gaza, Moskva: Izd.-vo MGU, 1989.

25. Dirak, P. A. M., Principy kvantovoj mehaniki, Moskva: Nauka, 1979.

26. Grim, G., Spektroskopija plazmy, Moskva: Atomizdat, 1969.

27. Sobel'man, I. I., Vvedenie v teoriju atomnyh spektrov, Moskva: Fiz.-mat. lit., 1963.

28. Arnold, J. O., Whiting, E. E., and Lyle, G. C., "Line by line calculation of spectra from diatomic molecules and atoms assuming a voigt line profile," J. Quant. Spectrosc. Radiat. Transf., Vol. 9, No. 6, Jun. 1969, pp. 775-798.

29. Kamenshhikov, V. A., Plastinin, Ju. A., Nikolaev, V. M., Novickij, L. A., Radiacionnye svojstva gazov pri vysokih temperaturah, Moskva: Mashinostroenie, 1971.

30. Vajnshtejn, L. A., Sobel'man, I. I., Jukov, E. A., Vozbuzhdenie atomov i ushirenie spektral'nyh linij, Moskva: Nauka, 1979.

31. Pener, S. S., Kolichestvennaja i molekuljarnaja spektroskopija i izluchatel'naja sposobnost' gazov, Moskva: Izd. Inostrannoj literatury, 1963.

32. “TOPbase - baza dannyh po sechenijam fotoionizacii.".

33. Glass, I. I. and Liu, W. S., "Effects of hydrogen impurities on shock structure and stability in ionizing monatomic gases," J. Fluid Mech., Vol. 84, No. 1, 1978, pp. 55-77. 